\title{
Comparison of choroidal thickness measurements between spectral-domain OCT and swept-source OCT in normal and diseased eyes
}

This article was published in the following Dove Press journal:

Clinical Ophthalmology

I4 November 2016

Number of times this article has been viewed

\author{
Sidra Zafar' \\ MA Rehman Siddiqui ${ }^{2,3}$ \\ Rida Shahzad' \\ 'Medical College, Aga Khan \\ University Hospital, ${ }^{2}$ Department of \\ Ophthalmology, Shahzad Eye Hospital, \\ ${ }^{3}$ South City Hospital, Karachi, Pakistan
}

Correspondence: MA Rehman Siddiqui Department of Ophthalmology, Shahzad Eye Hospital, B-2, Block-16, Gulshan-elqbal, Karachi, Pakistan

Tel +92 32l 2894547

Email rehman.siddiqui@gmail.com
Purpose: Sub-foveal choroidal thickness (SFCT) is affected in many ocular diseases. The aim of this study was to compare SFCT measurements between Topcon 3D 2000 spectral-domain optical coherence tomography (SD-OCT) and Topcon swept-source OCT (SS-OCT), with different laser wavelengths, in normal and diseased populations.

Materials and methods: This was a prospective, cross-sectional, noninterventional study including 27 normal volunteers and 27 participants with retinal disease. OCT scans were performed sequentially and under standardized conditions using both SD-OCT and SS-OCT. The OCT scans were evaluated by two independent graders. Paired $t$-tests and intraclass correlation coefficients (ICCs) were used to assess the statistically significant difference between SFCT measurements as measured by the two devices.

Results: Mean SFCT measurements for all 54 participants were 264.9 $\pm 103.1 \mu \mathrm{m}$ using SDOCT (range: $47-470 \mu \mathrm{m}$ ) and $278.5 \pm 110.5 \mu \mathrm{m}$ using SS-OCT (range: 56-502 $\mu \mathrm{m}$ ), with an inter-device ICC of 0.850 . Greater variability was noted in the diseased eyes. Inter-device ICCs were 0.870 (95\% CI; 0.760-0.924) and 0.840 (95\% CI; 0.654-0.930) for normal and diseased eyes, respectively. However, the difference was not statistically significant $(P=0.132)$.

Conclusion: Both machines reliably measure SFCT. Larger studies are needed to confirm these findings.

Keywords: choroidal imaging, diseased, normal, SD-OCT, SS-OCT

\section{Introduction}

The choroid is a thin, highly vascularized layer between the sclera and retina, which plays an important role in ocular metabolism and temperature regulation. ${ }^{1,2}$ Choroidal changes such as thickening, thinning and hyperpermeability are known to play a role in the pathophysiology of various ocular diseases including age-related macular degeneration (ARMD), glaucoma, central serous chorioretinopathy and pathological myopia. ${ }^{3}$ Therefore, evaluation of sub-foveal choroidal thickness (SFCT) as well as information about topographical variation of choroidal thickness in various diseases is important in understanding their pathophysiology. Traditional modalities such as ultrasonography, because of their limited resolution and low repeatability, have limited role in the assessment of the choroid..$^{4,5}$

A significant development in ocular imaging was the introduction of optical coherence tomography (OCT) that allowed high-quality imaging of ocular structures. The enhanced depth imaging (EDI) protocol was first introduced by Spaide et $\mathrm{al}^{6}$ in 2008 that allowed better visualization of the choroid. ${ }^{5,6}$ They showed that by placing the zero-delay line closer to the eye, an inverted image of the choroid may be produced. 
However, the enhanced choroidal resolution comes at the expense of decreased retinal clarity.

Swept-source OCT (SS-OCT) uses a longer wavelength of $1,050 \mathrm{~nm}$ laser that allows deeper penetration because of low light scattering. In addition, the ability of SS-OCT to accommodate more A-scans per frame results in a much faster rate of image acquisition. Faster sweeping rates decrease imaging artifacts due to eye movements and also allow evaluation of a large area/volume. Commercially available SS-OCT machines are also capable of creating automatic choroidal volumes and thickness measurements, which was not possible with spectral-domain (SD-OCT) machines. ${ }^{3,7,14}$

The aim of this study was to compare the differences in SFCT measurements between SD-OCT and SS-OCT in normal and diseased eyes.

\section{Materials and methods}

This was a prospective, cross-sectional, noninterventional study carried out at Shahzad Eye Hospital, Karachi, Pakistan, from May 2015 to September 2015. The hospital's ethics review committee reviewed and approved the study. All the procedures performed in this study were in accordance with the ethical standards of the institutional research committee and the Declaration of Helsinki. Written informed consent was obtained from all the participants. A total of 54 participants were included, comprising normal volunteers and patients with posterior segment diseases.

For normal participants, the inclusion criteria were an age range of 20-60 years, normal visual acuity and a normal fundus on OCT examination. The exclusion criteria were history of ocular and systemic diseases and prior intraocular surgery or intraocular injections.

The OCT devices compared in our study were as follows: 1) SD-OCT machine (Topcon 3D 2000; Topcon Inc., Tokyo, Japan) and 2) SS-OCT machine (Triton; Topcon Inc.). The SS-OCT has a scanning speed of 100,000 A-scans/sec. Using a wavelength of $1,050 \mathrm{~nm}$, it can penetrate deeper, visualizing ocular tissues such as the choroid or even the sclera. In comparison, the conventional SD-OCT utilizes a wavelength of $850 \mathrm{~nm}$ and has an image acquisition rate of 50,000 A-scans/sec.

All scans were performed by a single trained operator under standardized conditions and at a fixed time range (14:00-18:00) to reduce the influence of diurnal variation on choroidal thickness. All participants underwent sequential scanning by the two machines at the same sitting. The machines were used in random order. Right eye scans were performed first. Scans were read by trained medical staff.
A standardized imaging protocol was followed for all scans. For manual estimation of choroidal thickness, line scans were performed on both SD-OCT and SS-OCT. The single"line" protocol averages $50 \mathrm{~B}$-scans and generates a $6 \mathrm{~mm}$ line on the SD-OCT device and a $12 \mathrm{~mm}$ line with $96 \mathrm{~B}$-scans on the SS-OCT device. Only on the SS-OCT device, an additional $7 \times 7$ scanning protocol was performed. This was used for automatic choroidal thickness measurements.

Acquired images were required to have a minimum score of 45. Poor quality images that did not meet expectations were immediately repeated. SFCT was defined as a perpendicular distance from the lower border of retinal pigment epithelium-Bruch's membrane complex to the sclerochoroidal interface (SCI), which was measured sub-foveally. SFCT was measured independently by two graders using built-in caliper tool available on both the OCT devices. Automatic choroidal thickness measurements were ascertained using the built-in software on the SS-OCT device.

For statistical analysis, one eye of each patient was selected. In normal volunteers, this was performed randomly. In patients with ocular diseases, the more severely affected eye or the one with active disease was chosen. SPSS 20 (SPSS Inc., Chicago, IL, USA) was used for statistical analysis. The differences in choroidal thickness between the two machines were analyzed using paired $t$-tests. A $P$-value $<0.05$ was considered statistically significant. Intraclass correlation coefficients (ICCs) with 95\% confidence interval (CI) were used to assess intra-grader, intergrader and inter-device reliability.

\section{Results}

A total of 54 eyes of 54 patients were evaluated, of which 24 were right eyes (12 normal and 12 diseased). The mean age of all the participants was $42.6 \pm 14.7$ years (range: $20-75$ years) and 32 were male (Table 1). A total of 27 normal volunteers and 27 patients with retinal disease were included; nine had diabetic macular edema, seven had ARMD, four had epiretinal membranes, three had cystoid macular edema, two had central serous retinopathy (CSR) and one each with full-thickness macular hole and rhegmatogenous retinal detachment.

Evaluating all 54 eyes using manual measurements, the mean SFCT was calculated as $264.9 \pm 103.1 \mu \mathrm{m}$ using SD-OCT (range: $47-470 \mu \mathrm{m}$ ) and $278.5 \pm 110.5 \mu \mathrm{m}$ using SS-OCT (range: 56-502 $\mu \mathrm{m}$ ). However, the differences in choroidal thickness measured by SD-OCT and SS-OCT were not statistically significant $(P=0.132)$. Intra-reader and inter-reader reliability coefficients were high for both devices. Intra-reader ICCs for normal eyes were 0.988 
Table I Demographics of included participants and the frequency of diseases present in the diseased subgroup of 27 eyes

\begin{tabular}{ll}
\hline Patient characteristics & Frequency $(\mathbf{n})$ \\
\hline Age (mean \pm standard deviation, years) & $42.6 \pm 14.7$ \\
Gender & \\
Female & 22 \\
Male & 32 \\
Total patients & 54 \\
Normal & 27 \\
Retinal pathology & 27 \\
DME & 9 \\
ARMD & 7 \\
ERM & 4 \\
CME & 3 \\
CSR & 2 \\
Macular hole & 1 \\
RRD & 1 \\
\hline
\end{tabular}

Abbreviations: ARMD, age-related macular degeneration; CME, cystoid macular edema; CSR, central serous retinopathy; DME, diabetic macular edema; ERM, epiretinal membrane; RRD, rhegmatogenous retinal detachment.

(95\% CI: 0.981-0.993) and 0.966 (95\% CI: 0.948-0.979) for SD-OCT, and 0.992 (95\% CI: 0.987-0.995) and 0.985 (95\% CI: 0.975-0.992) for SS-OCT. For diseased eyes, intra-reader ICCs were 0.957 (95\% CI: 0.918-0.980) and 0.956 (95\% CI: $0.916-0.978$ ) for SD-OCT and 0.988 (95\% CI: 0.980-0.993) and 0.998 (95\% CI: 0.996-0.999) for SS-OCT. Inter-reader ICCs for normal eyes were 0.920 (95\% CI: $0.862-0.953$ ) and 0.923 (95\% CI: 0.857-0.958) for SD-OCT and SS-OCT, respectively. In diseased eyes, inter-reader ICCs were 0.842 (95\% CI: $0.654-0.928)$ for SD-OCT and 0.90 (95\% CI: 0.790-0.953) for SS-OCT. Inter-device ICCs were 0.870 (0.760-0.924) and 0.840 (95\% CI: 0.654-0.930) for normal and diseased eyes, respectively (Table 2).
For normal participants, mean SFCT measured manually with SD-OCT was 331.54 $\pm 68 \mu \mathrm{m}$ (range; $175-470 \mu \mathrm{m}$ ) and with SS-OCT was $333.4 \pm 78.6 \mu \mathrm{m}(166-502 \mu \mathrm{m} ; P=0.857)$. Mean SFCT in diseased eyes was $198.33 \pm 85.8 \mu \mathrm{m}$ with the SD-OCT device (47-369 $\mu \mathrm{m})$ and $225.4 \pm 109.2 \mu \mathrm{m}$ with the SS-OCT device (56-492 $\mu \mathrm{m} ; P=0.136)$.

Mean automatic SFCT measured by SS-OCT in the normal subjects was $300.4 \pm 67.2 \mu \mathrm{m}$. In the diseased participants, it was measured at $183.8 \pm 99.9 \mu \mathrm{m}$. The ICCs for manual and automatic choroidal thickness using SS-OCT were 0.932 (95\% CI; 0.834-0.980) for normal eyes and 0.919 for diseased eyes $(0.828-0.962)$. Bland-Altman plots comparing choroidal thickness measurements for the normal and diseased groups are shown in Figure 1.

\section{Discussion}

The choroid is a highly vascularized structure that plays an essential role in ocular nourishment. ${ }^{1,2}$ It consists of five layers; the innermost Bruch's membrane, choriocapillaris, Sattler's layer, Haller's layer and SCI. ${ }^{8}$ Measuring choroidal thickness can be challenging because of the variability that exists with diurnal influences, age and ocular pathology. Although the use of SD-OCT with EDI technique improved the quality of choroidal OCT, ${ }^{6}$ the introduction of SS-OCT was a significant development for consistent visualization of deeper ocular structures. ${ }^{8}$ Various studies in recent years have used both SD-OCT and SS-OCT devices for comparison of choroidal thickness and reported good inter-observer and inter-device reproducibility. ${ }^{1,9-11,13}$

Table 2 Comparison of choroidal thickness measurements between SD-OCT and SS-OCT in normal and diseased eyes

\begin{tabular}{|c|c|c|c|c|}
\hline SFCT & SD-OCT & SS-OCT & $P$-value & $\begin{array}{l}\text { Inter-device } \\
\text { ICC }\end{array}$ \\
\hline \multicolumn{5}{|l|}{ Mean SFCT measured manually } \\
\hline Total participants $(\mathrm{n}=54)$ & $264.9 \pm 103.1 \mu \mathrm{m}$ & $278.5 \pm 110.5 \mu \mathrm{m}$ & $P=0.132$ & \\
\hline Normal subgroup $(n=27)$ & $331.54 \pm 68 \mu \mathrm{m}$ & $333.4 \pm 78.6 \mu \mathrm{m}$ & $P=0.857$ & \\
\hline Diseased subgroup $(n=27)$ & $198.33 \pm 85.8 \mu \mathrm{m}$ & $225.4 \pm 109.2 \mu \mathrm{m}$ & $P=0.136$ & \\
\hline \multicolumn{5}{|l|}{ ICCs } \\
\hline Normal subgroup $(n=27)$ & & & & 0.870 \\
\hline Intra-reader I & 0.988 & 0.992 & & \\
\hline Intra-reader 2 & 0.966 & 0.985 & & \\
\hline Inter-reader & 0.920 & 0.923 & & \\
\hline Diseased subgroup $(n=27)$ & & & & 0.840 \\
\hline Intra-reader I & 0.957 & 0.988 & & \\
\hline Intra-reader 2 & 0.956 & 0.998 & & \\
\hline Inter-reader & 0.842 & 0.900 & & \\
\hline \multicolumn{5}{|c|}{ Mean SFCT measured automatically } \\
\hline Normal subgroup $(\mathrm{n}=27)$ & & $300.4 \pm 67.2 \mu \mathrm{m}$ & & \\
\hline Diseased subgroup $(n=27)$ & & $183.8 \pm 99.9 \mu \mathrm{m}$ & & \\
\hline
\end{tabular}

Abbreviations: ICC, intraclass correlation coefficient; SD-OCT, spectral-domain optical coherence tomography; SFCT, sub-foveal choroidal thickness; SS-OCT, sweptsource optical coherence tomography. 


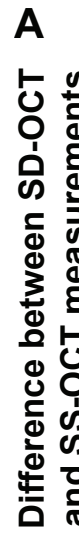

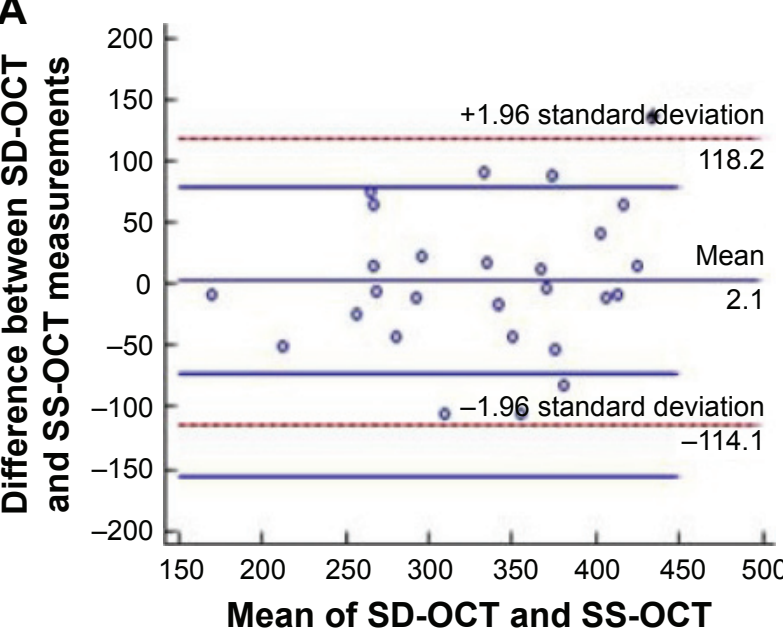

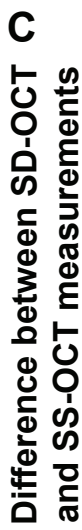

B

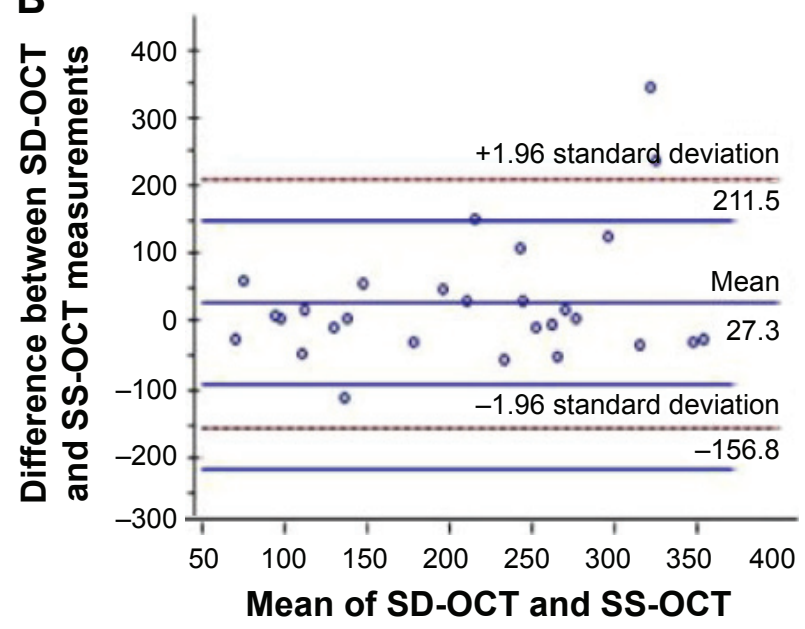

o +1.96 standard deviation
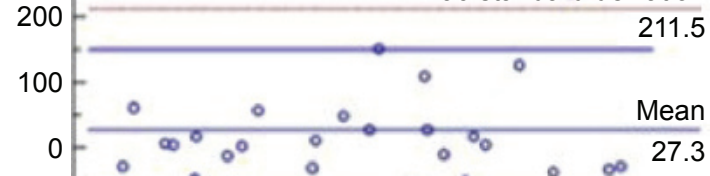

$-100-1.96$ standard deviation $-156.8$

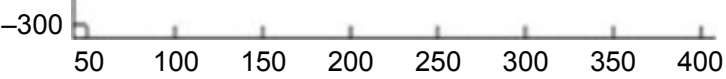

Mean of SD-OCT and SS-OCT

Figure I Bland-Altman plots.

Notes: (A) Comparison of choroidal thickness measurements between SD-OCT and SS-OCT in normal eyes. (B) Comparison of SD-OCT and SS-OCT in diseased eyes. (C) Comparison of SD-OCT with SS-OCT in all participants. The horizontal axis represents the mean of SD-OCT and SS-OCT measurements, and the vertical axis represents the difference between the measurements of SD-OCT and SS-OCT.

Abbreviations: SD-OCT, spectral-domain optical coherence tomography; SS-OCT, swept-source optical coherence tomography.

To the best of our knowledge, this is the first study comparing choroidal thickness between normal and diseased populations using SD-OCT and commercially available SS-OCT machines in a South Asian population. Our study is also unique because it compared automatic measurements of choroidal thickness with manual measurements in healthy and diseased participants.

Michalewski et $\mathrm{al}^{3}$ had earlier presented automatic choroid thickness measurements in a normal population (15-79 years). Choroidal thickness reported in their study with SS-OCT $(221 \mu \mathrm{m})$ was lower than the thickness measured manually $(259 \mu \mathrm{m})$. They postulated that the difference could be explained by the fact that the manual measurement is a focal measurement and that the automatic measurement measures the mean thickness in a circle with a diameter of $1,000 \mu \mathrm{m}$. Their study also reported a discrepancy in $16 \%$ of cases between the examiners' estimation and the automated measurement. Similarly, choroidal thickness measured automatically by SS-OCT in our diseased population was $184 \mu \mathrm{m}$, lower than that determined with manual measurements on either of the two devices. A possible reason for this could be automatic detection, and segmentation of the choroid by the software may not represent the actual SCI. This discrepancy was also noted by us while recording measurements. We noticed that SCI identified by the software was significantly higher in some participants. Automatic choroidal thickness measurement is a relatively new feature that became available with the introduction of SS-OCT machines. ${ }^{3,7,14}$ Further studies are thus required to assess its reliability especially in diseased populations, where a greater variation in choroidal thickness exists.

In a similar study, Tan et al ${ }^{1}$ measured choroidal thickness between normal and diseased populations using SDOCT (Spectralis SD-OCT; Heidelberg Engineering Inc., 
Heidelberg, Germany) and SS-OCT (Topcon DRI OCT-1; Topcon Inc.). They reported SD-OCT and SS-OCT choroidal thickness measurements of $272.4 \mu \mathrm{m}$ and $264.3 \mu \mathrm{m}$, respectively, for all 100 participants combined. At $264.9 \mu \mathrm{m}$ for SD-OCT and $279.5 \mu \mathrm{m}$ for SS-OCT, our results of choroidal thickness are comparable with their study. The differences in choroidal thickness between the two studies may be attributed to differences in age of the participants, ethnicity, axial length, severity of the disease process and perhaps diurnal influences. In our study, choroidal measurements were higher with SSOCT for both normal and diseased populations, a finding that was also reported by Matsuo et $\mathrm{al}^{9}(269.1 \mu \mathrm{m}$ for Topcon 3D OCT 1,000 versus $280.5 \mu \mathrm{m}$ for DRI OCT) and Ikuno et $\mathrm{al}^{10}$ in normal subjects $(283.7 \mu \mathrm{m}$ versus $292.7 \mu \mathrm{m})$. Matsuo et al hypothesized that the choroidal thickness measurements were thicker using SS-OCT, because the SCI seen on SDOCT scans may not be the true border. A similar discrepancy regarding the true border of SCI was also observed by us for SD-OCT images. SS-OCT images of the same eye, however, allowed visualization of the SCI more distinctly, therefore allowing more accurate measurements. This observation has also been made by other authors. ${ }^{12,13}$ Our study compared SD-OCT and SS-OCT among patients with retinal diseases. The results of our study demonstrated a larger variability in choroidal thickness measurement in diseased eyes compared with normal eyes, a finding consistent with Tan et al. ${ }^{1}$ Mean SFCT in the diseased group was $198.33 \pm 85.8 \mu \mathrm{m}$ with SDOCT and 225.4 $\pm 109.2 \mu \mathrm{m}$ with SS-OCT. Different variables may have contributed including disease severity causing greater variability between the graders when measuring the SFCT. Disease severity can also affect clear visibility of posterior boundaries, making it difficult to demarcate the SCI interface. We recommend choroidal thickness measurements for most retinal diseases. For example, even though most ARMD types show thin choroid, idiopathic polypoidal choroidal vasculopathy subtype shows a thicker choroid. Both conditions respond differently to anti-vascular endothelial growth factor (VEGF). Similarly, the late-onset chronic CSR, which mimics ARMD, shows thicker choroid. Treatment with half-fluence photodynamic therapy instead of anti-VEGF may show a markedly different response in this condition.

The strengths of our study include a sufficient sample, inclusion of only one eye per patient to reduce statistical variance and consistent evaluation of normal and abnormal eyes by the same graders.

\section{Conclusion}

Both SD-OCT and SS-OCT can measure choroidal thickness. SD-OCT requires switching to EDI mode before measurements can be recorded. SS-OCT gives less variability in choroidal thickness measurements particularly when the retinal architecture is abnormal such as that in diseased eyes. Larger studies are needed to confirm these findings.

\section{Abbreviations}

EDI, enhanced depth imaging; OCT, optical coherence tomography; SD, spectral domain; SS, swept source; SFCT, sub foveal choroidal thickness; SCI, sclerochoroidal interface.

\section{Acknowledgments}

The authors would like to acknowledge Mr Syed Iqbal Azam, Assistant Professor, Department of Community Health Sciences, Aga Khan University Hospital, Karachi, Pakistan, for his assistance with statistical analysis; Mr Adnan Sheikh, Shahzad Eye Hospital, Karachi, Pakistan, for his assistance with this project and Dr MH Shahzad, FRCS, MBBS, Consultant Ophthalmologist, Shahzad Eye Hospital, for the use of facilities at Shahzad Eye Hospital.

\section{Disclosure}

The authors report no conflicts of interest in this work.

\section{References}

1. Tan CS, Ngo W, Cheong KX. Comparison of choroidal thicknesses using swept source and spectral domain optical coherence tomography in diseased and normal eyes. Br J Ophthalmol. 2014;99(3): 354-358.

2. Feldkaemper MP, Wang HY, Schaeffel F. Changes in retinal and choroidal gene expression during development of refractive errors in chicks. Invest Ophthalmol Vis Sci. 2000;41(7):1623-1628.

3. Michalewski J, Michalewska Z, Nawrocka Z, Bednarski M, Nawrocki J. Correlation of choroidal thickness and volume measurements with axial length and age using swept source optical coherence tomography and optical low-coherence reflectometry. Biomed Res Int. 2014;2014: 639160 .

4. Mansouri K, Medeiros FA, Tatham AJ, Marchase N, Weinreb RN. Evaluation of retinal and choroidal thickness by swept-source optical coherence tomography: repeatability and assessment of artifacts. $\mathrm{Am}$ J Ophthalmol. 2014;157(5):1022-1032.

5. Mansouri K, Leite MT, Medeiros FA, Leung CK, Weinreb RN. Assessment of rates of structural change in glaucoma using imaging technologies. Eye. 2011;25(3):269-277.

6. Spaide RF, Koizumi H, Pozzoni MC. Enhanced depth imaging spectraldomain optical coherence tomography. Am J Ophthalmol. 2008;146(4): 496-500.

7. Ruiz-Medrano J, Flores-Moreno I, Pena-Garcia P, Montero JA, Duker JS, Ruiz-Moreno JM. Macular choroidal thickness profile in a healthy population measured by swept-source optical coherence tomography. Invest Ophthalmol Vis Sci. 2014;55(6):3532-3542.

8. Piasecka K, Michalewska Z. Choroidal imaging with swept source optical coherence tomography-a review. Eur Ophthalmic Rev. 2014;8(2): 132-136.

9. Matsuo Y, Sakamoto T, Yamashita T, Tomita M, Shirasawa M, Terasaki H. Comparisons of choroidal thickness of normal eyes obtained by two different spectral-domain OCT instruments and one swept-source OCT instrument. Invest Ophthalmol Vis Sci. 2013;54(12): $7630-7636$. 
10. Ikuno Y, Maruko I, Yasuno Y, et al. Reproducibility of retinal and choroidal thickness measurements in enhanced depth imaging and high-penetration optical coherence tomography. Invest Ophthalmol Vis Sci. 2011;52(8):5536-5540.

11. Copete S, Flores-Moreno I, Montero JA, Duker JS, Ruiz-Moreno JM. Direct comparison of spectral-domain and swept-source OCT in the measurement of choroidal thickness in normal eyes. Br J Ophthalmol. 2014;98(3):334-338.

12. Hirata M, Tsujikawa A, Matsumoto A, et al. Macular choroidal thickness and volume in normal subjects measured by swept-source optical coherence tomography. Invest Ophthalmol Vis Sci. 2011;52(8): 4971-4978.
13. Adhi M, Liu JJ, Qavi AH, et al. Choroidal analysis in healthy eyes using swept-source optical coherence tomography compared to spectral domain optical coherence tomography. Am J Ophthalmol. 2014; 157(6):1272-1281.

14. Michalewska Z, Michalewski J, Nawrocka Z, Dulczewska-Cichecka K, Nawrocki J. Suprachoroidal layer and suprachoroidal space delineating the outer margin of the choroid in swept-source optical coherence tomography. Retina. 2015;35(2):244-249.
Clinical Ophthalmology

\section{Publish your work in this journal}

Clinical Ophthalmology is an international, peer-reviewed journal covering all subspecialties within ophthalmology. Key topics include: Optometry; Visual science; Pharmacology and drug therapy in eye diseases; Basic Sciences; Primary and Secondary eye care; Patient Safety and Quality of Care Improvements. This journal is indexed on

\section{Dovepress}

PubMed Central and CAS, and is the official journal of The Society of Clinical Ophthalmology (SCO). The manuscript management system is completely online and includes a very quick and fair peer-review system, which is all easy to use. Visit http://www.dovepress.com/ testimonials.php to read real quotes from published authors. 